\title{
Pediatric Cardiovascular Device Registries-The Need for Such Data And The Potential Impact
}

\author{
Francesca Joseph
}

Received: 31 March 2010/Accepted: 12 May 2010/Published online: 29 June 2010

(C) Springer Science+Business Media, LLC 2010

The use of devices to treat disorders and diseases affecting pediatric patients has increased significantly since the 1980s, particularly in the field of pediatric cardiology where interventional procedures have become viable alternatives to open heart surgery for many conditions $[1,2]$. Yet as the treatment of congenital heart disease (CHD) with cardiac catheterization and device placement has evolved, there remains a lack of devices on the market that are designed for, studied in, and specifically labeled for pediatric patients and evidence-based data to guide decision making in treating these patients remains scarce. Treatment strategies instead are derived largely from expert opinionwhich may not be consistent across locales or widely accessible - and off-label use is not uncommon [3]. With the emphasis on evidence-based practice in medicine today, it is important that pediatrics not be left behind. If the present situation is to change, and the quality and consistency of information about catheter-based device treatment for pediatric cardiology patients is to improve, a method of collecting, analyzing, and widely distributing procedural data becomes essential. The development of more comprehensive pediatric device registries would thus seem a critical part of the progression and of utmost importance to the public health.

Registries provide opportunities to collect data from a broader population of patients and physicians than current surveillance mechanisms and could be used to monitor actual practices and practice bias for both on- and off-label use of devices used in children - better defining the current clinical landscape so that unmet needs and supportive

F. Joseph $(\bowtie)$

US FDA,

10903 New Hampshire Ave, Silver Spring, MD 20993, USA

e-mail: francesca.joseph@fda.hhs.gov regulatory initiatives can be more easily and quickly identified. Also, collection of long-term, longitudinal data on significant events, functionality, and quality of care that are so essential to optimizing pediatric care could be attained. The limited numbers of patients with any given congenital cardiac anomaly, and the heterogeneous subpopulations, makes pediatric cardiology an especially challenging area to obtain valid and generalizable data. Thus, it is one in which high-quality registry data would be particularly suitable.

\section{The Stakeholders}

As it stands now, the pediatric practitioner is often required to make decisions about device use and treatments based on limited information compared to the adult cardiologist. Though valid literature and guidelines do exist, the relative paucity of data is such that anecdotes and personal experience still form the basis for decisions in many cases. Registries with more comprehensive, accessible data could help to fill the gaps in evidence. Making data available on a national scale via a registry could reveal trends and help to identify clinical questions most appropriate for an Investigational Device Exemption, the results of which might contribute to the optimization and refinement of treatment guidelines. Furthermore, by making comparisons between centers possible with impunity, quality improvement initiatives could be facilitated across them and more consistent care could be brought to patients nationally.

From a regulatory perspective, the US Food and Drug Administration (FDA) routinely makes review decisions on permanent medical device implants designed to treat pediatric patients based on limited preapproval data with the requirement for postapproval long-term safety and 
effectiveness assessment. Yet today, few registries exist that are optimally designed to handle FDA postmarket surveillance challenges. Issues such as standard definitions, ability to link different databases, and risk-adjustments for the highly heterogeneous pediatric patient population remain.

Better, more numerous registries need not replace current postmarket surveillance systems, but certainly can be used to enhance them. They can increase reporting of devicerelated adverse events. They can provide the opportunity to develop standardized definitions, including definitions of risk factors related to devices and device applications that, when used across the field, would allow more meaningful comparisons across multiple trials and existing registries. And by including product-specific information in the context of patient disease management, registries can serve as valuable sources of data for assessing device performance in the postmarket setting as well as identify device performance trends and hypotheses for follow-up studies.

From a premarket perspective, FDA has faced difficulties in encouraging new device development for pediatric indications and in designing clinical studies to gain pediatric indications for devices approved or cleared for other indications. Often, with "off-label" device use as the standard of care, a study randomizing device treatment to medical or surgical therapy becomes difficult, if not impossible, to conduct. High quality clinical registries could provide valid reference data to enable more feasible premarket clinical trial designs, perhaps by using data from the registry to establish performance goals - point estimates to serve as a control.

For manufacturers, such databases can generate valueadded information on product use, market context, and outcomes [4]. Registries can serve as a system to identify and track devices intended for pediatric use and adult devices intended for conditions that also occur in children. The data could also be used to identify devices that would require minimal modifications or testing to obtain a pediatric indication. Although there is often a cost involved, the overall cost of data collection may be reduced by eliminating unnecessary redundancies that may exist among current systems.

\section{Progress}

Databases that collect data on cardiovascular use of medical devices do already exist-e.g., the Extracorporeal Life Support Organization registry, Congenital Cardiovascular Interventional Study Consortium — but momentum is building for projects with a more extensive outcome assessment goal for pediatric cardiology practices in the United States and abroad [5].

In the USA, the American College of Cardiology is a leader in such an effort. Partnering with the Society for
Cardiovascular Angiography and Interventions, a new, national clinical data registry called IMproving Pediatric and Adult Congenital Treatment (IMPACT ${ }^{\mathrm{TM}}$ ), is being developed under the auspices of the National Cardiovascular Data Registry [5]. To present, registries of CHD have been limited in scope - narrowed to specific CHD diagnoses or procedures. But CHD is not a limited disease, and thus demands an adequate assessment of the diversity of procedures and variety of management patterns that occur across its spectrum. Taking the approach of leveraging expertise in existing congenital cardiac disease registries and developing collaborations with national associations in an effort to help cardiologists measure performance and outcomes of procedures, design quality improvement initiatives, and improve quality of care for patients with congenital cardiac malformations, IMPACT ${ }^{\mathrm{TM}}$ would represent a significant step forward.

The project is backed by funding from the FDA and, as of the composition of this article (February 2010), the pilot phase is underway and the full registry set to launch in the next few months. The registry will assess the prevalence, demographics, management, and outcomes of pediatric and adult patients with congenital heart disease who are undergoing diagnostic catheterization and catheter-based interventions. Although initially the IMPACT Registry [6] will focus on episodes of care, eventually it is hoped to become a longitudinal database used to improve care not just around a procedure, but over the lifetime of the patient. Furthermore, the IMPACT data could potentially be applied to solve some of the regulatory issues noted above, and FDA involvement will help to ensure the collection of usable data that may eventually be used to advance the development and expedite the approval process for new safe and effective devices.

\section{Looking Ahead}

In the field of pediatric cardiology, where a lack of specifically labeled pediatric devices, off-label device use and sometimes undocumented outcomes are the status quo; data collected via registries becomes particularly valuable and urgently necessary. The clinical community, FDA, device manufacturers, and most of all patients, all stand to benefit from the information gained. As collaboration to enhance the pathways to approval of cardiac devices for children is underway, adding registry data to the effort seems an indispensable complement. With such a tool in hand, the pediatric cardiology field would be well poised to move quickly beyond outcome assessment and benchmarking, to collaborative quality improvement, as a "lifetime" for children with heart disease becomes ever longer.

The views and opinions are those of the author and do not necessarily reflect those of the US Food and Drug 
Administration, the US Department of Health and Human Services, or the Public Health Service.

\section{References}

1. Beekman, et al. (2009). Pathways to approval of pediatric cardiac devices in the United States: Challenges and solutions. Pediatrics, 124, e155-e162.

2. Hijazi, Z., \& Awad, S. (2008). Pediatric cardiac interventions. Journal of American College of Cardiology Interventional Scientific Council, 1, 603-611.
3. Sutherell, J. S., Hirsch, R., \& Beekman, R. H., 3rd. (2010). Pediatric interventional cardiology in the United States is dependent on the off-label use of medical devices. Congenital Heart Disease, 5(1), 2-7.

4. US FDA. Medical Devices. FDA Workshop: The role of clinical data in postmarket surveillance of cardiovascular devices. Available at: http://www.fda.gov/MedicalDevices/NewsEvents/Workshops Conferences/ucm114684.htm.

5. Jenkins, K., et al. (2008). Databases for assessing the outcomes of the treatment of patients with congenital and paediatric cardiac disease - The perspective of cardiology. Cardiology in the Young, 18(Suppl. 2), 116-123.

6. "IMPACT Registry". Available at http://impact.ncdr.com. Retrieved January 29, 2010. 